\title{
Surface proteomic analysis of differentiated versus stem-like osteosarcoma human cells
}

\author{
Marica Gemei ${ }^{1}$, Claudia Corbo ${ }^{1}$, Francesca D'Alessio ${ }^{1}$, Rosa Di Noto ${ }^{1,2}$, Renza Vento ${ }^{3}$ \\ and Luigi Del Vecchio ${ }^{1,2}$ \\ ${ }^{1}$ CEINGE-Biotecnologie Avanzate s.c.a.r.l., Napoli, Italy \\ ${ }^{2}$ Dipartimento di Medicina molecolare e Biotecnologie mediche, Università "Federico II" di Napoli, Napoli, Italy \\ ${ }^{3}$ Dipartimento di Scienze Biomediche, Università Degli Studi di Palermo, Policlinico, Palermo, Italy
}

Cancer stem cell characterization represents a breakthrough in cancer research. Despite evidence showing the existence and the role of cancer stem cells in osteosarcoma (OS) onset and progression, little is known about their specific surface phenotype. To address this issue, we carried out a cytometric analysis with an antibody-array comprising 245 membrane proteins comparing the stem and differentiated OS cells. As experimental model, we chose the stem-like cell line 3aminobenzamide-OS and its parental, differentiated, cell line MG63. We identified 50 differentially expressed, 23 homogeneously expressed, and 172 not expressed proteins in the two cell line models, thus defining a surface protein signature specific for each of them. Furthermore, we selected ERK1/2 (p44/42 mitogen-activated protein kinases) as a potential pathway correlated with processes that characterize tumorigenic potential and stemness of 3aminobenzamide-OS cells.

\section{Keywords:}

Cancer stem cell / Cell biology / Osteosarcoma / Surface proteome

Additional supporting information may be found in the online version of this article at the publisher's web-site

Osteosarcoma (OS) is the most frequent nonhematologic form of primary bone cancer in children and young adults, accounting for about $15-20 \%$ of all bone tumors [1,2]. It is a devastating malignant neoplasm arising from primitive transformed cells of mesenchymal origin exhibiting osteoblastic differentiation and that produces malignant osteoids [3]. Due to the aggressive malignant potential and early metastasis, OS is a disease with a low long-term survival rate and a poor early diagnosis. In fact, despite important progresses in surgery,

Correspondence: Dr. Marica Gemei, CEINGE Biotecnologie Avanzate s.c.a.r.l., Via Gaetano Salvatore 486, (ex Comunale Margherita, 482), 80145, Napoli, Italia

E-mail: maricagemei@gmail.com

Fax: +390813737808

Abbreviations: 3-AB, 3-aminobenzamide; $C D$, cluster of differentiation; CSC, cancer stem cell; ERK, extracellular signal-regulated kinase; IPA, ingenuity pathway analysis; MAPK, p44/42 mitogenactivated protein kinases; OS, osteosarcoma; PAK, p21-activated kinase; PTEN, phosphatase and tensin homologue deleted on chromosome 10
Received: May 2, 2013

Revised: July 23, 2013

Accepted: August 21, 2013 radiotherapy and neoadjuvant chemotherapy, the morbidness of OS has been increased by $1.4 \%$ per year in the past 25 years $[4,5]$. Increasing evidence suggests that tumors are organized in a hierarchy of heterogeneous cell populations, with the capability to sustain tumor formation and growth residing specifically in a small subset of cells, that is, the cancer stem cells (CSCs) [6-8]. These cells are endowed with both self-renewal and multilineage differentiation potential; they induce cell proliferation, invasion, and metastasis. Despite it is still unclear whether a CSC originates from transformed organ stem cells or whether it acquires the self-renewal capability during tumorigenic transformation, the existence of CSC and their role of "source" for tumor maintenance/growth is today widely accepted. CSCs confer resistance to therapies; thus, a successful cure of cancer should require CSCs eradication. Therefore, a number of recent studies have been focused on CSCs existence revelation and/or on the understanding of their features and molecular mechanisms in the attempt to

Colour Online: See the article online to view Fig. 1 in colour. 
Table 1. Surface proteins differentially expressed in $3 A B-O S$ and MG63 cells. Percentages of positive cells are reported for both cell lines

\begin{tabular}{|c|c|c|c|}
\hline Antigen & Gene symbol & 3AB-OS & MG63 \\
\hline CD1b & CD1b & 2.0 & bdl \\
\hline CD10 & CALLA & 8.9 & bdl \\
\hline CD30 & TNFRSF8 & 83.8 & bdl \\
\hline CD32 & FCGR2A & 0.7 & bdl \\
\hline CD49a & ITGA1 & 67.8 & bdl \\
\hline CD108 & SEMA7A & 45.0 & bdl \\
\hline CD138 & SDC1 & 78.1 & bdl \\
\hline CD141 & THBD & 14.5 & bdl \\
\hline CD146 & MCAM & 99.8 & bdl \\
\hline CD171 & L1CAM & 28.2 & bdl \\
\hline CD133 & PROM1 & 71.3 & bdl \\
\hline CD13 & ANPEP & bdl & 100 \\
\hline CD26 & DPP4 & bdl & 5.9 \\
\hline CD42A & GP9 & bdl & 13.8 \\
\hline CD42B & GP1BA & bdl & 29.2 \\
\hline CD49B & ITGA2 & bdl & 94.7 \\
\hline CD54 & ICAM1 & bdl & 28.5 \\
\hline CD62E & SELE & bdl & 3.3 \\
\hline CD95 & FAS & bdl & 98.1 \\
\hline CD91 & LRP1 & bdl & 22.1 \\
\hline CD99R & MIC2 receptor & bdl & 4.7 \\
\hline CD130 & IL6ST & bdl & 9.3 \\
\hline CD140A & PDGFRA & bdl & 93.0 \\
\hline CD140B & PDGFRB & bdl & 49.1 \\
\hline CD142 & F3 & bdl & 1.8 \\
\hline CD166 & ALCAM & bdl & 79.6 \\
\hline CD227 & MUC1 & bdl & 93.3 \\
\hline SSEA4 & SSEA4 & bdl & 16.8 \\
\hline CD243 & ABCB1 & 2.9 & 4.1 \\
\hline CD9 & CD9 & 80.2 & 99.3 \\
\hline CD47 & CD47 & 24.5 & 98.3 \\
\hline CD49C & ITGA3 & 29.0 & 99.7 \\
\hline CD51/61 & CD51/61 & 43.9 & 96.5 \\
\hline CD55 & DAF & 60.7 & 99.8 \\
\hline CD61 & ITGB3 & 1.9 & 53.8 \\
\hline CD77 & A4GALT & 7.9 & 62 \\
\hline CD99 & $\mathrm{MIC2}$ & 23.7 & 89.3 \\
\hline$\beta-2$ microglobulin & B2MICRO & 10.8 & 98.3 \\
\hline EGFR & EGFR & 66.5 & 94.8 \\
\hline HLAABC & HLAABC & 35.8 & 99.8 \\
\hline CD49F & ITGA6 & 12.2 & 60 \\
\hline CD340 & ERBB2 & 75.1 & 82.3 \\
\hline CD221 & IGF1R & 39.0 & 77.2 \\
\hline CD24 & CD24 & 13.8 & 5 \\
\hline CD97 & TM7LN1 & 13.0 & 4.9 \\
\hline CD56 & NCAM1 & 99.3 & 18.6 \\
\hline CD165 & CD165 & 98.6 & 3.9 \\
\hline CD271 & NGFR & 84.5 & 12.4 \\
\hline MICA,MICB & MICA,MICB & 95.5 & 2.5 \\
\hline CD164 & CD164 & 37.1 & 83.5 \\
\hline
\end{tabular}

bdl: below detection limit.

open the way for the development of new targeted therapies for malignant tumors [9-17].

The revelation of a subpopulation of stem-like cells in OS dates back to 2005 by Gibbs et al. [18], after that several research groups addressed their studies on isolation and identification of OS stem cells using various methods [19-24].

Recently, Di Fiore et al. induced the selection of a cancer stem-like population from the MG63 OS cell line. This new cell line has been named 3-aminobenzamide(AB)-OS since it has been selected and stabilized by treating human OS MG63 cells with 3-AB, a potent competitive inhibitor of poly(ADPribose)- polymerase, for about 100 days [25].

The $3 \mathrm{AB}-\mathrm{OS}$ is a stable and immortalized CSC line that represents the stem component of MG63 parental cell line. Differently from MG63 cells, 3AB-OS cells can grow unlimitedly without losing their proliferative potential [25]. They showed a high drug efflux capacity dependent on adenosine triphospate binding-cassette G2 transporters. 3AB-OS showed a strong positivity for the stem cell marker CD133, which was almost immeasurable in MG63 cells [25]. Moreover $3 \mathrm{AB}-\mathrm{OS}$ cells were much less committed to osteogenic and adipogenic differentiation than MG63 cells and displayed a high expression of the genes required for maintaining stem cell state (Octamer-binding transcription factor 3/4, human telomerase reverse transcriptase, nucleostemin, Nanog) and for inhibiting apoptosis (hypoxia-inducible factor-1a, B cell lymphoma-2, and survivin) [25]. Gene expression profile of 3AB-OS in comparison with MG63 revealed the differential regulation of 196 genes involved in important pathways connected to tumorigenesis and stemness, namely Wnt, Notch, Hedgehog, and extracellular signal-regulated kinase (ERK)1/2 (p44/42 mitogen-activated protein kinases, MAPK) signaling [26]. The 3AB-OS cells were highly tumorigenic in mice and tumors that they formed expressed, in a first time, high levels of pAKT, $\beta 1$-integrin, and pFAK, nuclear $\beta$ catenin, c-myelocytomatosis oncogene cellular homolog, cyclin D2, along with high levels of anti-apoptotic proteins such as B cell lymphoma-2 [27]. Engrafted tumor cells also showed multilineage commitment with matrigel particularly favoring the mesenchymal lineage. Concomitantly, many blood vessels and muscle fibers appeared in the tumor mass [27].

To better characterize the biochemical phenotype of $3 \mathrm{AB}$ OS CSCs, we have analyzed their specific surface proteome profile in comparison with the parental cell line MG63. We performed a high-throughput immunophenotype screening of the two cell lines using an antibody array (Lyoplate ${ }^{\circledR}$, Becton Dickinson, Franklin Lakes, NJ, USA) containing quite all "cluster of differentiation" (CD) surface molecules along with other surface antigens associated with stem cells phenotype (242 different specificities), moreover we added an other three antigens lacking in the original array (e.g. CD133, CD324, CD66c). Cells were grown in DMEM supplemented with $10 \% \mathrm{FBS}$ and $2 \%$ L-glutamine at a concentration of $20 \times 10^{3}$ cells $/ \mathrm{cm}^{2}$. When they reached confluence, cells were detached from culture flasks by trypsin treatment and washed with PBS to be stained. All primary antibodies were lyophilized and allocated into three 96-well plates. Antibodies were reconstituted as described by original datasheet and added to polystyrene tubes containing $100 \times 10^{3}$ cells each for both 3AB-OS and MG63 cell lines. Cells were incubated for $1 \mathrm{~h}$ at 


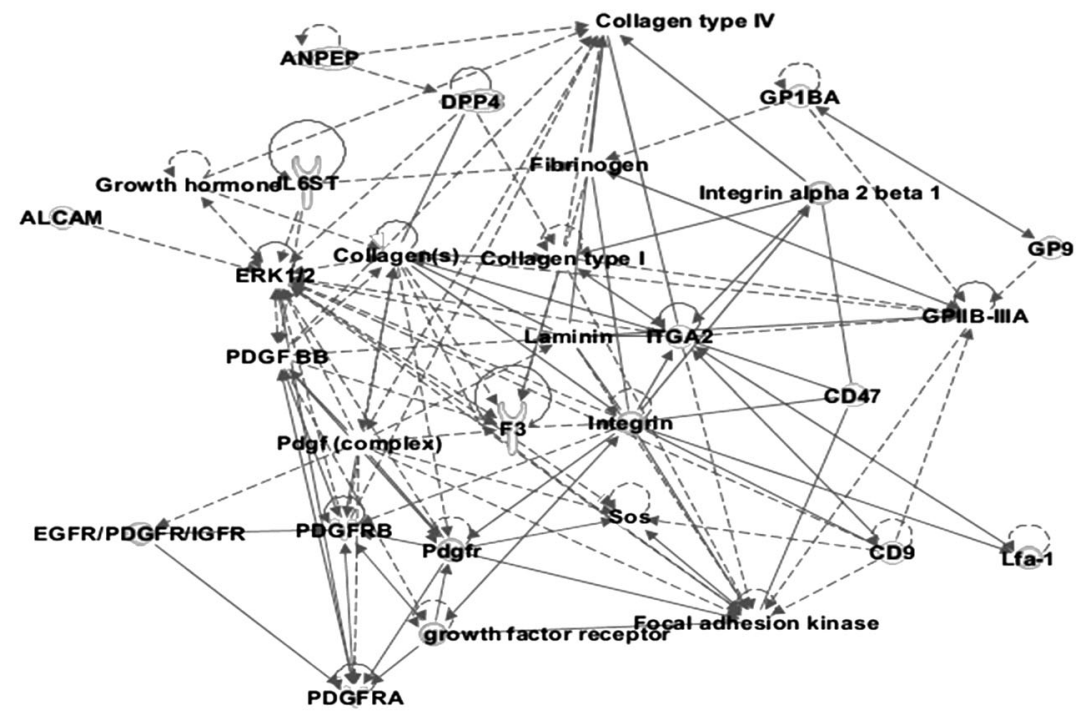

Figure 1. IPA network "cell-tocell signalling and interaction and inflammatory response." Proteins in gray were identified in this study, proteins in white were not identified but are reported to be involved in the network.

$4^{\circ} \mathrm{C}$ in the dark and then washed twice with $500 \mu \mathrm{L}$ of PBS $/ 2 \%$ FBS. Alexa Fluor 647-conjugated antimouse and antirat Ig secondary antibodies were diluted as described by the producer datasheet and added to cell pellets. Secondary antibodies were incubated for $30^{\prime}$ at $4^{\circ} \mathrm{C}$ in the dark. Cells were then washed twice with $500 \mu \mathrm{L}$ of PBS/2\% FBS and resuspended in $200 \mu \mathrm{L}$ of PBS/2\% FBS. All tubes were acquired at Beckmann Coulter FC500 cytometer. We analyzed a total of 245 surface specificities (Table 1 and Supporting Information Tables 1 , 2 , and 3) in triplicate experiments. Nine isotypic controls, five for antimouse and four for antirat primary antibodies (mouse IgM, IgG1, IgG2a, IgG2b, IgG3 and rat IgM, IgG1, IgG2a, IgG2b) were included in the Lyoplate and have been used as negative controls in the analysis. We have identified three groups of antigens: differentially expressed (50 proteins), homogeneously expressed (23 proteins), and homogeneously negative antigens (172 proteins; Supporting Information Tables 1 and 2). Pathways and cellular functions associated with differentially expressed antigens were analyzed by ingenuity pathway analysis (IPA) software 9.0 (http://www.ingenuity.com). IPA software creates networks of direct and indirect relationships between genes and proteins on the basis of literature data. To each network IPA associates a score computed according to Fisher's exact test that indicates the likelihood that interactions are random. The parameters setting we used in our analysis has been previously described [28]. The software generated a network (score 30) related to "cell-to-cell signaling and interaction and inflammatory response" (Fig. 1). Interestingly several proteins in the network were associated with tumor suppressors p21activated kinase and phosphatase and tensin homolog deleted on chromosome 10 canonical pathways (Fig. 2A). The cytometric dot plots of some of the differentially expressed surface proteins associated with p21-activated kinase and phos- phatase and tensin homolog deleted on chromosome 10 are shown in Supporting Information Fig. 1.

Most of the proteins involved in the two pathways are common; particularly a central role in both signaling is played by ERK1/2 (MAPK). MAPKs are involved in several cellular programs as cell proliferation, differentiation, motility and cell survival. Moreover, MAPKs signaling pathway is an important target in the diagnosis and treatment of cancer [29]. To confirm the involvement of MAPKs signaling in 3AB-OS cells, we decided to investigate the level of expression of ERK1/2 (MAPK) and its phosphorylated counterparts by Western blotting analysis (Fig. 2B and supplemental methods).

Our data demonstrated an activation of MAPKs pathway in 3AB-OS cells due to the exclusive presence of the phosphorylated form of MAPKs in this cell line (Fig. 2B). MAPKs pathway is involved in the cytoskeleton rearrangement during tumoral cells invasion processes suggesting that it could be connected to the previously observed enhanced motility and invasiveness of 3AB-OS cancer stem-like cells [25].

Our data reveal for the first time a significant number (245 antigens) of identified surface proteins in MG63 and in its derived CSC line 3AB-OS, thus highlighting a surface protein signatures specific differentiated MG63 parental cell line and its stem-like counterpart. Moreover, we associated the surface protein profile of 3AB-OS CSCs with MAPKs pathway activation, the latter was already found in 3AB-OS CSCs during transcriptome analysis performed by Di Fiore et al. [26].

We are confident that these data will benefit further investigations regarding this newly established cell line as well as ongoing research on OS CSCs. These findings enrich our knowledge of the surface proteome of OS stem cell, providing preliminary information also in the development of new 


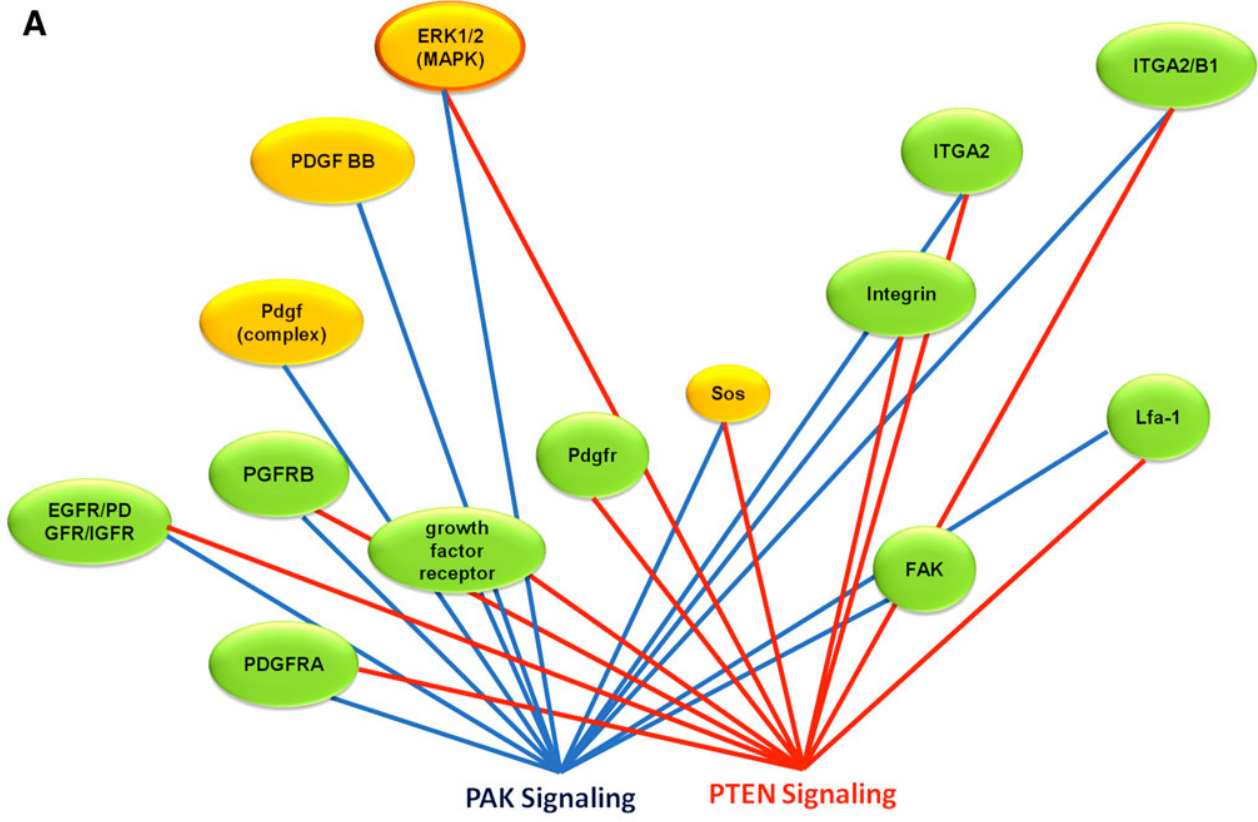

B

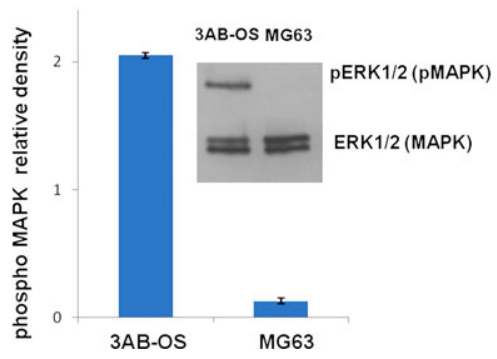

Figure 2. (A) PAK (p21-activated kinase) and PTEN (phosphatase and tensin homologue deleted on chromosome 10) pathways. Some of the proteins in the network "cell-to-cell signaling and interaction and inflammatory response" are associated with PAK (blue) and PTEN (red) canonical pathways. Molecules we identified differentially expressed are in green while those in orange were added by IPA network analysis. ERK $1 / 2$ (MAPK) is one of the central nodes of both PAK and PTEN pathways. (B) ERK1/2 (MAPK) protein expression level and phosphorylation status. Western blotting analysis and densitometric measurements of protein lysates of $3 A B-O S$ and MG63 cells. Proteins were separated on 4-15\% SDS-PAGE and immunoblotted with specific antibodies. The analyses were performed on three independent samples; the results are shown as mean $\pm \mathrm{SD}$. therapeutic approaches. In fact, surface protein profiling is a key step in the screening for new therapeutic targets; moreover surface antigens are suitable for cancer cell targeted monoclonal antibodies-based therapies.

This work was supported by grant from the Italian Association for Cancer Research AIRC (grant 10737 to professor Del Vecchio).

The authors have declared no conflict of interest.

\section{References}

[1] Picci, P., Osteosarcoma (osteogenic sarcoma). Orphanet. J. Rare Dis. 2007, 2, 6-10.

[2] Ottaviani, G., Jaffe, N., The epidemiology of osteosarcoma. N. Cancer Treat Res. 2009, 152, 3-13.

[3] Tang, N., Song, W. X., Luo, J., Haydon, R. C. et al., Osteosarcoma development and stem cell differentiation. Clin. Orthop. Relat. Res. 2008, 466, 2114-2130.

[4] Caudill, J. S., Arndt, C. A., Diagnosis and management of bone malignancy in adolescence. Adolesc. Med. State Art. Rev. 2007, 18, 62-78.
[5] Li, J. P., Liu, L. H., Li, J., Li, H. et al., Microarray expression profile of long noncoding RNAs in human osteosarcoma. Biochem. Biophys. Res. Commun. 2013, 433, 200-206.

[6] Clarke, M. F., Dick, J. E., Dirks, P. B., Eaves, C. J. et al., Cancer stem cells-perspectives on current status and future directions: AACR workshop on cancer stem cells. Cancer Res. 2006, 66, 9339-9344.

[7] Dalerba, P., Cho, R. W., Clarke, M. F., Cancer stem cells: models and concepts. Annu. Rev. Med. 2007, 58, 267-284.

[8] Shibata, M., Shen, M. M., The roots of cancer: stem cells and the basis for tumor heterogeneity. Bioessays 2013, 35, 253-260.

[9] Corbo, C., Orrù, S., Gemei, M., Di Noto, R. et al., Protein cross-talk in CD133+ colon cancer cells indicates activation of the Wnt pathway and upregulation of SRp20 that is potentially involved in tumorigenicity. Proteomics 2012, 12, 20452059.

[10] Gemei, M., Mirabelli, P., Di Noto, R., Corbo, C. et al., CD66c is a novel marker for colorectal cancer stem cell isolation, and its silencing halts tumor growth in vivo. Cancer 2013, 119, 729-738.

[11] Maitland, N. J., Collins, A. T., Prostate cancer stem cells: a new target for therapy. J. Clin. Oncol. 2008, 26, 2862-2870. 
[12] Al-Hajj, M., Clarke, M. F., Self-renewal and solid tumor stem cells. Oncogene 2004, 23, 7274-7282.

[13] Eramo, A., Lotti, F., Sette, G., Pilozzi, E. et al., Identification and expansion of the tumorigenic lung cancer stem cell population. Cell Death Differ. 2008, 15, 504-514.

[14] O' Brien, C. A., Pollett, A., Gallinger, S., Dick, J. E. et al., A human colon cancer cell capable of initiating tumour growth in immunodeficient mice. Nature 2007, 445, 106-110.

[15] Hu, Y., Yu, X., Liu, S., Liu, S., Cancer stem cells: a shifting subpopulation of cells with stemness? Med. Hypotheses 2013, $80,649-655$.

[16] Zhang, H., Li, S., Molecular mechanisms for survival regulation of chronic myeloid leukemia stem cells. Protein Cell 2013, 4, 186-96.

[17] Qian, X., Wagner, S., Ma, C., Klussmann, J. P. et al., ALDH1-positive cancer stem-like cells are enriched in nodal metastases of oropharyngeal squamous cell carcinoma independent of HPV status. Oncol. Rep. 2013, 29, 1777-1784.

[18] Gibbs, P. C., Kukekov, V. G., Reith, J. D., Tchigrinova, O. et al., Stem-like cells in bone sarcomas: Implications for tumorigenesis. Neoplasia 2005, 7, 967-976.

[19] Liu, B., Ma, W., Jha, R. K., Gurung, K., Cancer stem cells in osteosarcoma: recent progress and perspective. Acta Oncol. 2011, 50, 1142-1150.

[20] Martins-Neves, S. R., Lopes, Á. O., do Carmo, A., Paiva, A. A. et al., Therapeutic implications of an enriched cancer stemlike cell population in a human osteosarcoma cell line. BMC Cancer 2012, 12, 139-155.

[21] Wang, L., Park, P., Zhang, H., La Marca, F. et al., BMP-2 inhibits the tumorigenicity of cancer stem cells in human osteosarcoma OS99-1 cell line. Cancer Biol. Ther. 2011, 11, 457-463.

[22] Gong, C., Liao, H., Wang, J., Lin, Y. et al., LY294002 induces G0/G1 cell cycle arrest and apoptosis of cancer stem-like cells from human osteosarcoma via down-regulation of PI3K activity. Asian Pac. J. Cancer Prev. 2012, 13, 3103-3107.

[23] Zhang, H., Wu, H., Zheng, J., Yu, P. et al., Transforming growth factor $\beta 1$ signal is crucial for dedifferentiation of cancer cells to cancer stem cells in osteosarcoma. Stem Cells 2013, 31, 433-446.

[24] Liu, B., Ma, W., Jha, R. K., Gurung, K., Cancer stem cells in osteosarcoma: recent progress and perspective. Acta Oncologica 2011, 50, 1142-1150.

[25] Di Fiore, R., Santulli, A., Ferrante, R. D., Giuliano, M. et al., Identification and expansion of human osteosarcomacancer-stem cells by long-term 3-aminobenzamide treatment. J. Cell Physiol. 2009, 219, 301-313.

[26] Di Fiore, R., Fanale, D., Drago-Ferrante, R., Chiaradonna, F. et al., Genetic and molecular characterization of the human osteosarcoma 3AB-OS cancer stem cell line: a possible model for studying osteosarcoma origin and stemness. $J$. Cell Physiol. 2013, 228, 1189-1201.

[27] Di Fiore, R., Guercio, A., Puleio, R., Di Marco, P. et al., Modeling human osteosarcoma in mice through $3 A B-O S$ cancer stem cell xenografts. J. Cell Biochem. 2012, 113, 3380-3392.

[28] Caterino, M., Corbo, C., Imperlini, E., Armiraglio, M. et al., Differential proteomics analysis in human cells subjected to ribosomal stress. Proteomics 2013, 13, 1220-1227.

[29] Roberts, P. J., Der, C. J., Targeting the Raf-MEK-ERK mitogenactivated protein kinase cascade for the treatment of cancer. Oncogene 2007, 26, 3291-3310. 